\title{
Simplified Biological Tissue Analysis with Combination of Atmospheric Scanning Electron Microscope and Light Microscope
}

\author{
Akiko Hisada $^{1}$ and Yusuke Ominami ${ }^{2}$ \\ ${ }^{1}$ Hitachi Central Research Laboratory, Saitama, Japan \\ ${ }^{2}$ Hitachi High-Technologies Corporation, Ibaraki, Japan
}

Correlative light and electron microscopy has recently been considered to be a powerful tool for analyzing cell biology. For any observation that uses different types of microscopes in succession, specimens should be processed appropriately. Viable cells or fixed cells maintained in liquid can be observed with a light microscope. When those cells are successively observed with a conventional electron microscope, they should be processed so that they remain intact when placed in a high vacuum and irradiated with an electron beam. However, cells easily change shape if processed using drying. To keep the focus on cell samples that are processed differently, various procedures to create a trace of the visual field under a light and electron microscope have been proposed.

Another effective approach is to use an isolated atmospheric specimen chamber with an electron microscope. This device can prevent the deformation of cells caused by drying, making it easier to maintain the same field of view. Several types of specimen chambers have been designed for scanning electron microscopes (SEMs) [1-4].

To this end, we have developed a novel atmospheric scanning electron microscope (ASEM) that enables bulky biological specimens to be observed without preparations such as dehydration, drying, and electroconductive processing [5, 6]. In this study, we evaluated the ASEM images of individual sections of rat organs to establish an observation procedure combining ASEM and light microscopy.

An inner specimen chamber combined with a thin membrane enabling electron beam penetration is inserted in the main chamber of a desktop type SEM (figure 1, a and b). A specimen inside the inner chamber is kept at ambient atmospheric pressure (figure 1, b) or negative pressure (figure 1, c). Electron beam dispersion with the gas can be reduced by adjusting the negative pressure [6].

Rat organs were excised and fixed with neutral buffered formalin. After washing with water, sections were examined under an optical stereo microscope, and then identical sections were observed with ASEM. To evaluate the ASEM images, sections were processed with conventional H-E staining. As a result of ASEM observation, it was possible to visualize the cells of hydrated tissues without staining (figure 2). For example, we observed goblet cells of the intestinal mucosa surface, enterocytes of a mucosa section, and cartilage cavities and chondrocytes of a hyaline cartilage of the trachea.

We have established an observation procedure to directly examine a specimen under ASEM after making an optical stereo microscope observation without additional preparation. In conclusion, observing a section of the hydrated bulky tissue at a cellular level was simplified. Furthermore, we propose a new workflow to determine points of interest by making ASEM observations prior to timeconsuming histological analysis (figure 2). 
References:

[1] Danilatos GD, Scanning 7 (1985), p. 26.

[2] Thiberge S et al., Proc Natl Acad Sci USA. 101 (2004), p. 3346.

[3] Ogura T, Biochem Biophys Res Commun. 377 (2008), p. 79.

[4] Suga M et al., Ultramicroscopy 111 (2011), p. 1650.

[5] Ominami Y et al., Proc SPIE, 9236 (2014), p. 923604.

[6] Ominami Y et al., Microscopy, Epub 2014 Dec 23.

(a)

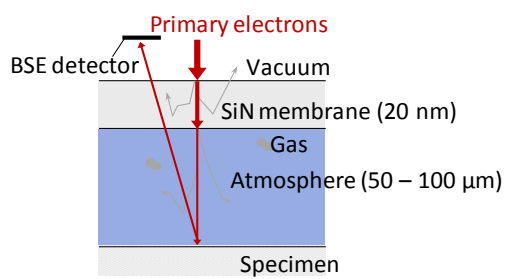

(b)

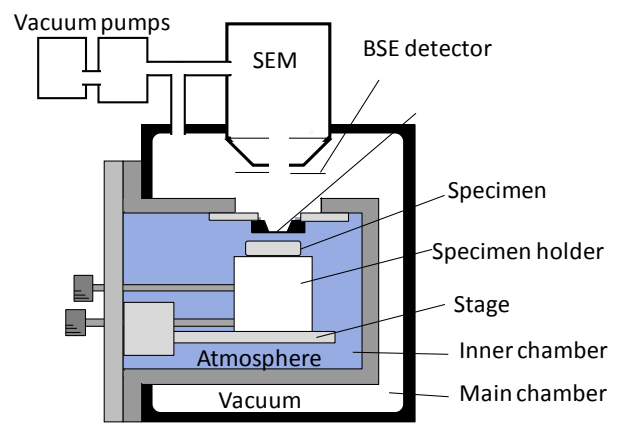

(c)

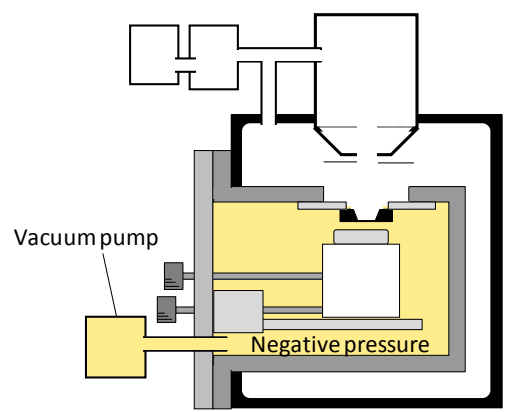

Figure 1. Schematic view of desktop type ASEM.

(a) Primary electrons penetrating membrane.

(b) Imaging mode at atmospheric pressure. Inner chamber is kept at $10^{5} \mathrm{~Pa}$ (blue).

(c) Negative pressure from $10^{3} \mathrm{~Pa}$ to $10^{5} \mathrm{~Pa}$ (yellow).

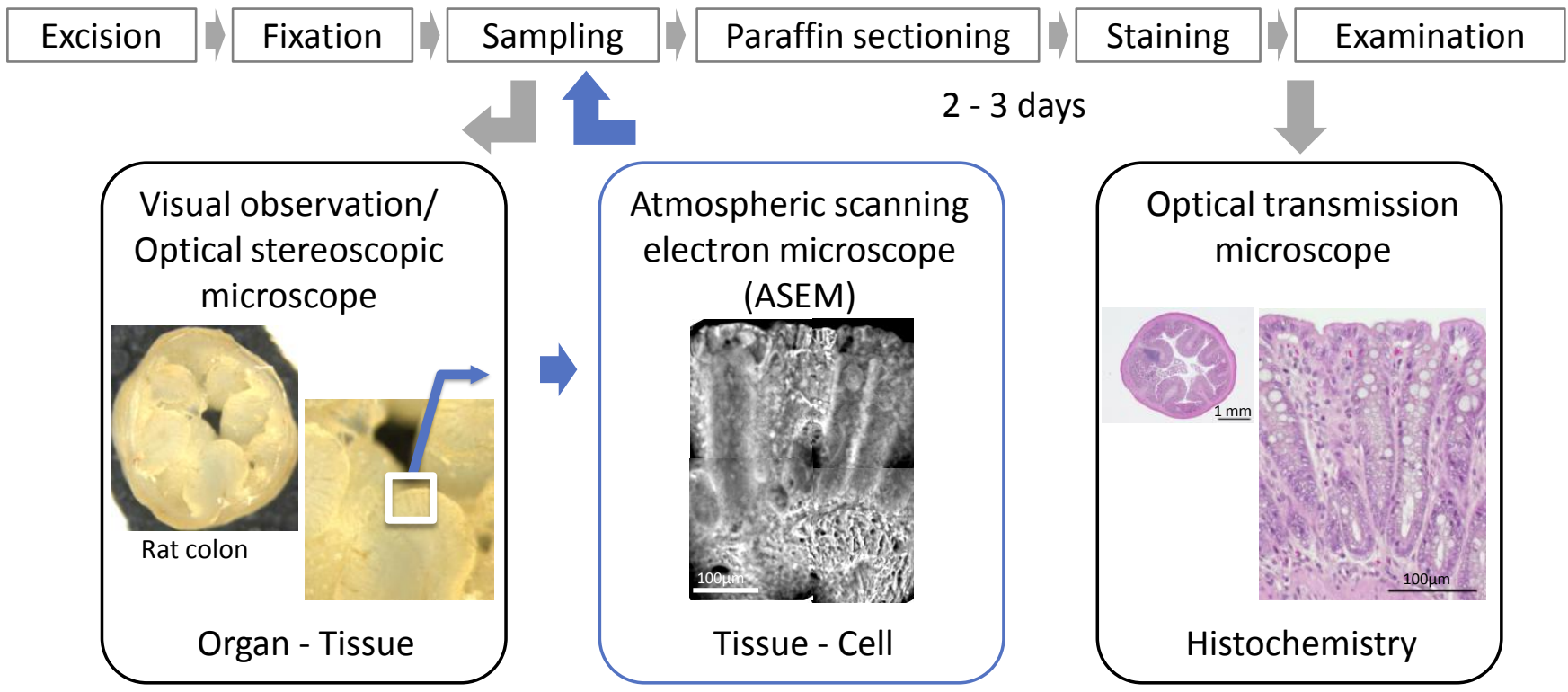

Figure 2. Proposed workflow for processing a piece of hydrated tissue for histological analysis. 